\title{
From cloud crash to star birth: star formation in cloud collisions
}

\author{
Kazuhiro Shima ${ }^{1}$, Elizabeth J. Tasker, and Asao Habe \\ Department of Physics, Faculty of Science, Hokkaido University, \\ Kita-ku, Spporo, 060-0810, Japan \\ ${ }^{1}$ email: shima@astro1.sci.hokudai.ac.jp
}

\begin{abstract}
We performed three-dimensional hydrodynamical simulations of idealized giant molecular cloud collisions including star formation and radiative transfer. We found that the characteristics of the colliding systems are similar to the observations of the Spitzer bubbles, suggesting these objects could be created in such interactions. A high velocity collision creates a top-heavy core mass distribution but is not strongly affected by radiation. At lower collision speeds, the HII regions have time to expand within the shock and promote the formation of massive cores.
\end{abstract}

Keywords. ISM: clouds, ISM: structure, stars: formation, methods: numerical

\section{Introduction}

We know that low mass stars can form via gravitational core collapse. However, this process can fail to produce very massive stars because the radiation pressure from the pre-stellar core can throttle accretion. To avoid the such problem, attention has been diverted to the role of collisions between the star-forming giant molecular clouds (GMCs). In this process, a turbulent shock wave forms at the collision interface. This can cause the surface density to become sufficiently high for the ram pressure to exceed the radiation barrier.

Observations and simulations now suggest cloud-cloud collisions could explain the formation of our most massive stars and super star clusters (e.g. Takahira et al. (2014), Torii et al. (2015), Haworth et al. (2015)). Due to their size, massive stars and super star clusters produce strong energy feedback, which affects the efficiency of the future star formation or may even stop it completely. Understanding this feedback effect is important because it is one of the controlling factors for the star formation rate in the galaxy.

In this work, we focus on the collision structure and the production and effect of HII bubbles on star formation using three-dimensional hydrodynamical simulations

\section{Numerical methods}

Our simulations were run with the adaptive mesh refinement (AMR) hydrodynamics code, Enzo. The limiting resolution (smallest cell size) during the runs is $\Delta x \simeq 0.015$ pc. A star particle forms when the cell density exceeds $10^{4} \mathrm{~cm}^{-3}$, forming a star cluster that has an assumed Salpeter IMF. The resulting cluster emits UV radiation using an adaptive ray-tracking scheme with a hydrogen ionising luminosity of $3 \times 10^{46} \mathrm{ph} / \mathrm{s} / \mathrm{M}_{\odot}$, where we assumed half the particle mass was converted into stars.

The colliding clouds were represented as unequally sized gas spheres with a Bonnor-Ebert density profile. The smaller cloud has a mass of $7.6 \times 10^{3} \mathrm{M}_{\odot}$ and a radius of $14 \mathrm{pc}$ while the larger cloud has a mass of $2.7 \times 10^{4} \mathrm{M}_{\odot}$ and a radius of $28 \mathrm{pc}$. The clouds were given additional internal support from an initial injection of turbulence. The simulations compare collisions with relative velocities of $10 \mathrm{~km} / \mathrm{s}, 20 \mathrm{~km} / \mathrm{s}$ and $30 \mathrm{~km} / \mathrm{s}$. 


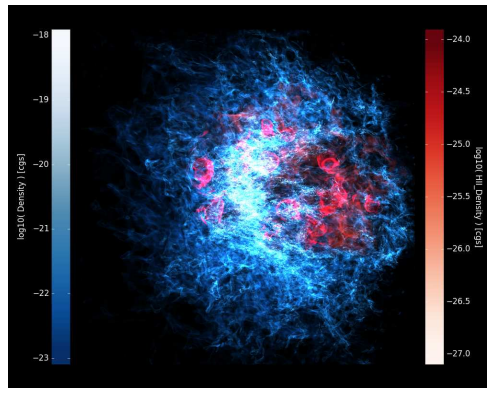

Figure 1. Volume rendering of gas density (blue) and HII density (red) in the $20 \mathrm{~km} / \mathrm{s}$ collision speed case at 4.0 Myr. The angle between the line-of sight and the collision axis is 45 degree.

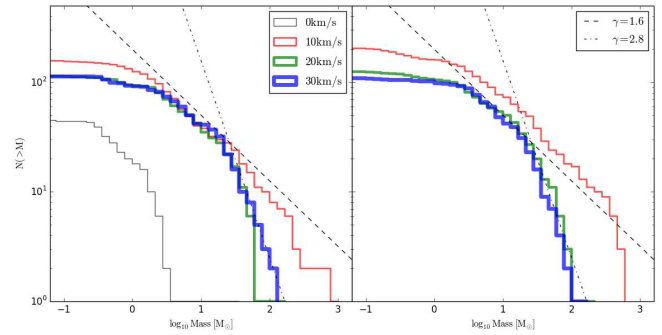

Figure 2. Cumulative core mass distributions (CMDs) after the crossing time, red $(10 \mathrm{~km} / \mathrm{s}): 6 \mathrm{Myr}$, green $(20 \mathrm{~km} / \mathrm{s}): 3 \mathrm{Myr}$, blue $(30 \mathrm{~km} / \mathrm{s}): 2 \mathrm{Myr}$, without feedback (left panel) and with feedback (right panel). The black solid line shows the CMD of the isolated cloud case after half the free-fall time.

\section{Results}

Fig. 1 shows a volume rendered view of the total gas density (blue) and HII density (red) in the $20 \mathrm{~km} / \mathrm{s}$ collision speed case at 4.0 Myr. The unequally sized clouds collide to produce a curved bow shock that forms a layer of dense material. The stars that form from this dense material emit radiation to create HII bubbles that fill the inner low density region behind the shock front. This hollowed-out shape resembles observations of the Spitzer bubbles (for example RCW120), suggesting that such a collision process could create these structures, not the alternative view of strong feedback from supernovae.

The panels in Fig. 2 show the cumulative core mass distributions (CMDs) after the collision crossing time: red $(10 \mathrm{~km} / \mathrm{s}): 6 \mathrm{Myr}$, green $(20 \mathrm{~km} / \mathrm{s}): 3 \mathrm{Myr}$, blue $(30 \mathrm{~km} / \mathrm{s}): 2 \mathrm{Myr}$. The left panel shows the collisions without any stellar feedback while the right panel shows the effect of including radiation.

During the collision, massive cores form with the number reaching about 100 in every collision case. For the high collision speed cases, we see a tighter bend in the CMD curve. This is due to the short time the shock spends within the cloud, reducing the time a forming core can accrete. The feedback has the strongest impact in the low velocity $(10 \mathrm{~km} / \mathrm{s})$ case, where the shock moves sufficiently slowly to allow the HII region to within the shock, boosting the shock strength. The velocity of the feedback-induced expansion is $\sim 7 \mathrm{~km} / \mathrm{s}$, corresponding to a sound speed with temperature $10^{4} \mathrm{~K}$. This is faster than the speed of the shock front which is $\sim 5 \mathrm{~km} / \mathrm{s}$ in the case of $10 \mathrm{~km} / \mathrm{s}$ collision. This increases the shock strength and triggers more star formation. In the faster collision cases, the HII region expands behind the shock front, where the two effects can not combine. The result is minimal change, with the star formation still being predominately controlled by the collisional shock.

\section{References}

Takahira, K., Tasker, E.J., \& Habe, A., 2014, ApJ, 792, 63

Torii, K., Hasegawa, K., Hattori, Y., Sano, H., Ohama, A., Yamamoto, H., Tachihara, K., Soga, S., Shimizu, S., Okuda, T. Mizuno, N., Onishi, T., Mizuno, A., \& Fukui, Y., 2015, ApJ, 806,7

Haworth, T.J., Tasker, E.J., Fukui, Y., Torii, K., Dale, J.E., Shima, K., Takahira, K., Habe, A., \& Hasegawa, K., 2015, MNRAS, 450, 10 